\title{
Z(2)-symmetric center vortex model with a first-order deconfinement transition
}

\section{Engelhardt* and B. Sperisen}

Department of Physics, New Mexico State University, Las Cruces, NM 88003, USA

E-mail: engel @nmsu. edu

\begin{abstract}
A random vortex world-surface model for the infrared sector of $S p(2)$ Yang-Mills theory is constructed. The $S p(2)$ gauge group, while allowing for the same set of center vortex fluxes as the $S U(2)$ gauge group, induces a significantly different dynamics on those vortex fluxes, which manifests itself in a first-order deconfinement phase transition. As shown by the construction presented here, a new vortex effective action term which can be interpreted in terms of a vortex stickiness can be used to drive the deconfinement transition towards first-order behavior. The available data from lattice $S p(2)$ Yang-Mills theory are reproduced quantitatively and, in addition, predictions for the behavior of the spatial string tension at high temperatures are presented.
\end{abstract}

XXIVth International Symposium on Lattice Field Theory

July 23-28, 2006

Tucson, Arizona, USA

${ }^{*}$ Speaker. 


\section{Introduction}

Recently, efforts have been undertaken to gain further insight into confinement mechanisms by studying Yang-Mills theories based on gauge groups other than $S U(N)$. In particular, the $G(2)$, $S p(2)$ and $S p(3)$ groups have been studied in detail [1-4]. One interesting comparison made possible by that work is the one between the $S p(2)$ and the $S U(2)$ cases: Both of these groups have the same center and also induce the same set of center vortex degrees of freedom in the respective Yang-Mills theories ${ }^{1}$. Nevertheless, $S U(2)$ Yang-Mills theory displays a second-order deconfinement phase transition, whereas the $S p(2)$ case displays a first-order transition. This observation, among others, led to the conclusion in [2-4] that the center plays no role in determining the order of the deconfinement phase transition, which is instead decisively influenced by the size of the gauge group.

Formulated slightly more precisely, the conclusion which can be drawn from the aforementioned work is that knowledge of the center of a gauge group alone does not permit a prediction of the order of the deconfinement transition. However, it should not be misconstrued as implying that the center degrees of freedom of the theory are irrelevant for the physics of confinement and the deconfinement transition. Indeed, knowledge of the set of center vortex degrees of freedom contained in a Yang-Mills theory does not suffice to specify its infrared effective vortex description and the associated infrared phenomenology; one of course must also give the effective action governing those vortex degrees of freedom. Only then is the infrared description complete. Vortex models based on the same set of degrees of freedom, but with different effective actions, can lead to vastly different physics. Accordingly, in the present investigation, an effective vortex model for the infrared sector of $S p(2)$ Yang-Mills theory is constructed. While based on the same set of vortex degrees of freedom as the $S U(2)$ model investigated in [5-7], it displays a first-order deconfinement phase transition, contrary to the latter case. Thus, the vortex picture is perfectly consistent with the $S U(2)-S p(2)$ comparison highlighted above; in the vortex language, integrating out the (differently sized) cosets in $S U(2)$ and $S p(2)$ Yang-Mills theory yields different effective actions for the respective vortex degrees of freedom. These different effective actions lead, in particular, to different behavior at the deconfinement transition. From this point of view, thus, the physics of confinement and, in particular, the deconfinement transition is still determined by infrared effective center vortex dynamics; the distinction between the $S U(2)$ and $S p(2)$ vortex models arises at the level of the specific effective actions inherited from the full gauge groups.

\section{Lattice Yang-Mills data}

The study [2] provides two relevant confinement characteristics of $S p(2)$ Yang-Mills theory, namely, the ratio of the deconfinement temperature to the square root of the zero-temperature string tension, $T_{c} / \sqrt{\sigma}$, and the latent heat at the transition $L_{H}$. The latter corresponds to the discontinuity in the four-dimensional action density ${ }^{2} \bar{s}$ at the first-order deconfinement transition, and is given

\footnotetext{
${ }^{1}$ Note that the center vortex fluxes allowed for by a gauge group $G$ are determined by the first homotopy group of the gauge group after factoring out its center $Z_{G}$, i.e., $\Pi_{1}\left(G / Z_{G}\right)$. In more physical terms, specifically for $S U(2)$ and all $S p(N)$ groups, the chromomagnetic flux carried by center vortices is quantized such as to contribute a center phase $(-1)$ to any Wilson loop to which it is linked.

${ }^{2}$ Since the symbol $s$ will be put to a different use further below, the action density is denoted by $\bar{s}$ in the following.
} 
in [2] in lattice units, i.e., $L_{H}=a^{4} \Delta \bar{s}$, where $a$ denotes the lattice spacing. It should be noted that [2] only gives $L_{H}$ quantitatively at one rather strong coupling, $8 / g^{2}=6.4643$; on the other hand, $T_{c} / \sqrt{\sigma}$ is available for a number of couplings, including the extrapolation to the continuum limit. The most consistent way to model the available data is to aim at reproducing $S p(2)$ YangMills theory specifically at the coupling $8 / g^{2}=6.4643$, at which both $L_{H}$ and $T_{c} / \sqrt{\sigma}$ are known (rather than using input from two different couplings, i.e., $8 / g^{2}=6.4643$ in the one case and the continuum limit in the other).

For $8 / g^{2}=6.4643$, the ratio of the deconfinement temperature to the square root of the zerotemperature string tension is [2]

$$
T_{c} / \sqrt{\sigma}=0.59 .
$$

On the other hand, identifying $L_{H}=a^{4} \Delta \bar{s}$, one has [2]

$$
N_{t}\left(a^{4} \Delta \bar{s}\right)^{2} / 4=0.15
$$

for the action density discontinuity $\Delta \bar{s}$, where $N_{t}$ denotes the extent of the lattice in the (Euclidean) time direction. Combining this with the fact that, at $8 / g^{2}=6.4643$, the deconfinement transition occurs at $N_{t}=2$, i.e., the deconfinement temperature satisfies $a T_{c}=1 / 2$, the lattice spacing can be eliminated, resulting in

$$
\Delta \bar{s} / T_{c}^{4}=8.76 .
$$

Relations (2.1) and (2.3) are used as input data for the random vortex world-surface model to be constructed in the following.

\section{Random vortex world-surface model}

Random vortex world-surface models describe the infrared, strongly interacting regime of Yang-Mills theories on the basis of effective gluonic center vortex degrees of freedom. A description in terms of such degrees of freedom was initially suggested and studied in [8-12]; compelling motivation for this picture is provided by more recent investigations of the relevance of center vortices in the lattice Yang-Mills ensemble [13 - 18], for a review, cf. [19]. Random vortex worldsurface models have been investigated both with respect to $S U(2)$ as well as $S U(3)$ Yang-Mills theory $[5-7,20-22]$. They successfully reproduce the main features of the strongly interacting vacuum. In the $S U(2)$ case, not only has a confining low-temperature phase been obtained together with a second-order deconfinement phase transition as temperature is raised [5]; also the topological susceptibility [6, 23-25] and the (quenched) chiral condensate [7] of $S U$ (2) Yang-Mills theory are reproduced quantitatively. In the $S U(3)$ case, the deconfinement transition becomes weakly first order [20] and a Y-law for the baryonic static potential results in the confining phase [21].

Recent efforts have concentrated on exploring the range of applicability of random vortex world-surface models as the Yang-Mills gauge group is varied. An investigation of the confinement properties in a vortex model for $S U$ (4) Yang-Mills theory [26] revealed signatures of Abelian magnetic monopoles, which are an intrinsic feature of generic center vortex configurations due to vortex world-surface non-orientability, beginning to influence the vortex dynamics for $S U(4)$ color. Contrary to the $S U(2)$ and $S U(3)$ cases, the vortex action cannot be expressed purely in terms of world-surface properties anymore, but acquires contributions attributable to the monopoles. Such 
a shift in the dynamical characteristics is expected as the number of colors rises [27]; the model construction presented in [26] explicitly confirms that expectation. The thrust of the present investigation is similar in nature, as already discussed further above: To be able to model the infrared sector of $S p(2)$ Yang-Mills theory, new features need to be introduced into the effective vortex action.

A practical computational framework for random vortex world-surface models is achieved by composing vortex world-surfaces of elementary squares on a hypercubic space-time lattice ${ }^{3}$. An ensemble of random world-surfaces is generated by Metropolis Monte Carlo update, where the closed nature of the surfaces (implied by the Bianchi constraint on the chromomagnetic fields) is preserved by simultaneously updating all six sides of an elementary three-dimensional cube at each step. The weighting of world-surface configurations is determined by an action of the symbolic form

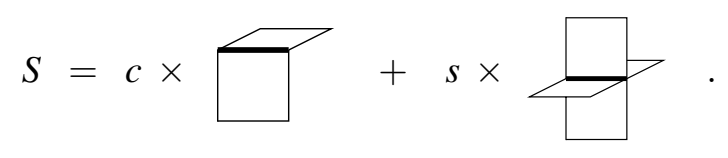

The first of these two action terms is a curvature term: Each instance of two vortex elementary squares sharing a link without lying in the same plane costs an action increment $c$, thus penalizing vortices "going around a corner". For the $S U(2)$ and $S U(3)$ cases studied in [5-7, 20-22], this single term was sufficient to achieve a phenomenologically viable model for the infrared sector of the corresponding Yang-Mills theory.

By contrast, to arrive at a model for the infrared sector of $S p(2)$ Yang-Mills theory, additional dynamics must be introduced; otherwise, the finite-temperature deconfinement phase transition remains second order. The second term in (3.1) can be interpreted as follows: Each instance of more than two vortex elementary squares being attached to a link is weighted ${ }^{4}$ by an action increment $s$. Thus, for negative coefficient $s$, vortex world-surfaces are encouraged to intersect along whole lines in space-time. This means that, when two vortex flux lines meet, they tend to maintain their contact over longer distances. In this sense, the second action term in (3.1) can be viewed as implementing a "stickiness" of the vortices. This choice of action is motivated by the experience with the $S U(4)$ random vortex world-surface model [26]. Also in that case, it is necessary to enhance the first-order character of the deconfinement phase transition. This is achieved by facilitating vortex branching, a process possible in the $S U(4)$ case due to the existence of two types of center flux. While the $S p(2)$ case discussed here does not allow for branching since there is only one type of center flux, the stickiness term in (3.1) is similar to a branching term in that it encourages more than two vortex elementary squares being attached to a link (for negative $s$ ). For this reason, such an action term seems a promising choice, which indeed is vindicated by the numerical results described below.

\section{Locating the physical point}

Having generated a random vortex world-surface ensemble according to the model defined

\footnotetext{
${ }^{3}$ The lattice spacing in this approach is a finite physical quantity implementing the notion that vortices possess a finite transverse thickness and must be a minimal distance apart to be distinguished from one another.

${ }^{4}$ This includes both the case of four as well as the case of six elementary squares being attached to the link. In general, one can associate these two cases with different action increments; however, throughout the present investigation, they were weighted, ad hoc, with the same action increment.
} 
above, one can measure the action density and the string tension (using the fact that Wilson loops acquire a center phase $(-1)$ for every instance of a vortex piercing an area spanned by the loop). Since the lattice spacing is a finite physical quantity, only discrete temperatures can be realized at any given set of coupling parameters $c$ and $s$ in (3.1). In general, therefore, the deconfinement phase transition is not directly accessible at the physical set of coupling parameters. Instead, one studies several unphysical sets of $c, s$ which do realize the transition (as evidenced by a double peak in the action density distribution) on lattices extending a varying number $N_{t}$ of spacings in the (Euclidean) time direction. The gathered data can then be interpolated to obtain, e.g., the latent heat at the physical point in the space of coupling constants. The simplest such interpolation scheme is obtained by using $N_{t}=1,2$, equivalent to $a T_{c}=1$ and $a T_{c}=0.5$; Table 1 displays corresponding data taken at suitable coupling parameters $c, s$.

\begin{tabular}{|c||c|c|c|c|}
\hline$a T_{c}$ & $c$ & $s$ & $\Delta \bar{s} / T_{c}^{4}$ & $T_{c} / \sqrt{\sigma}$ \\
\hline \hline 1 & 0.3394 & -1.24 & 0.014 & 0.816 \\
\hline 0.5 & 0.5469 & -1.99 & 13 & 0.474 \\
\hline
\end{tabular}

Table 1: Sets of coupling parameters $c, s$ realizing the deconfinement phase transition on lattices with $N_{t}=1,2$, together with measurements of the latent heat and the ratio of the deconfinement temperature to the square root of the zero-temperature string tension.

Note that the requirement of realizing the deconfinement transition alone of course does not fix both $c$ and $s$ simultaneously; the particular sets of coupling parameters quoted in Table 1 were singled out by the additional requirement that linear interpolation of the data must yield the physical point, at which both the relations (2.1) and (2.3) are satisfied. Indeed, linearly interpolating $\Delta \bar{s} / T_{c}^{4}$ as a function of $T_{c} / \sqrt{\sigma}$, one obtains $\Delta \bar{s} / T_{c}^{4}=8.76$, as required by (2.3), for $T_{c} / \sqrt{\sigma} \approx 0.59$, cf. (2.1). Likewise, linearly interpolating $c$ and $s$ as functions of $T_{c} / \sqrt{\sigma}$ yields the physical set of coupling parameters

$$
c=0.479 \quad s=-1.745 .
$$

Finally, one can also interpolate $a T_{c}$ as a function of $T_{c} / \sqrt{\sigma}$, which yields

$$
a T_{c}=0.663
$$

at the physical point. Thus, contrary to the $S U(N)$ vortex models $[5,20,26]$, the physical point in the present case is not near one of the measured data sets. In all $S U(N)$ cases studied, the physical point is very near the $N_{t}=2$ data set, and the uncertainty engendered by the interpolation is consequently very small. Here, by contrast, the uncertainty inherent in the interpolation is substantial and it is useful to consider consistency checks. One simple such check can be made as follows: While deconfinement transition data are not directly accessible at the physical point, the zero-temperature string tension $\sigma$ is. One can measure $\sigma a^{2}$ directly for the physical set of couplings (4.1) and combine this with (4.2) to obtain an additional determination of $T_{c} / \sqrt{\sigma}$. This indeed again yields $T_{c} / \sqrt{\sigma}=0.59$, buttressing the interpolation procedure used above. Another possibility, which will be reported in detail elsewhere [28], lies in using a larger data set, with $N_{t}=1,2,3$, for the interpolation. This yields deviations of the order of $10 \%$ in the determination of the physical point, giving another indication of the amount of uncertainty. It should, however, 
be kept in mind that using $N_{t}=1,2,3$ is not necessarily more accurate than just using $N_{t}=1,2$ data; after all, $N_{t}=3$ is rather far removed from the physical point and may thus easily introduce systematic distortions into the results rather than rendering them more accurate.

Finally, having determined the physical set of coupling parameters (4.1), one can predict further nonperturbative characteristics of $S p(2)$ Yang-Mills theory, such as the behavior of the spatial string tension $\sigma_{S}$ at high temperatures. Corresponding measurements for different $N_{t}$ using (4.1) are displayed in Table 2, where $N_{t}$ has been translated into $T / T_{c}$ using (4.2).

\begin{tabular}{|c||c|c|c|}
\hline$T / T_{c}$ & 0.50 & 0.75 & 1.51 \\
\hline \hline$\sigma_{S}(T) / \sigma_{S}(T=0)$ & 1.00 & 1.02 & 1.36 \\
\hline
\end{tabular}

Table 2: Predictions for the behavior of the spatial string tension $\sigma_{S}$ at high temperatures.

These predictions can be used to test the validity of the present random vortex world-surface model construction by comparing with corresponding measurements in $S p(2)$ lattice Yang-Mills theory.

\section{Conclusions}

By introducing a vortex "stickiness" into the action of an effective $Z(2)$-symmetric random vortex world-surface model, it is possible to drive its finite-temperature deconfinement phase transition towards first-order behavior. This is necessary if such a model in particular is to replicate the infrared phenomenology of $S p(2)$ Yang-Mills theory. Indeed, within the present investigation, it proved possible to reproduce available data from lattice $S p(2)$ Yang-Mills theory quantitatively by appropriate choice of the curvature and stickiness coupling parameters.

This successful reconstruction of $S p(2)$ Yang-Mills confinement characteristics within the random vortex world-surface model underscores that the $S U(2)-S p(2)$ comparison discussed at the outset does not contradict the vortex picture of Yang-Mills vacuum dynamics; while the two gauge groups indeed engender the same vortex topology, they lead to different vortex effective actions (induced by integrating out their differently sized cosets), and thus naturally to different behavior at the deconfinement phase transition.

In addition, the random vortex world-surface model constructed here yields predictions for the behavior of the spatial string tension at high temperatures, cf. Table 2. Comparison with corresponding measurements within $S p(2)$ lattice Yang-Mills theory could be used to further test the validity of the model.

\section{Acknowledgments}

This work was supported by the U.S. DOE under grants DE-FG03-95ER40965 (M.E.) and DE-FG02-94ER40847 (B.S.).

\section{References}

[1] K. Holland, P. Minkowski, M. Pepe, and U.-J. Wiese, Nucl. Phys. B668, 207 (2003). 
[2] K. Holland, M. Pepe, and U.-J. Wiese, Nucl. Phys. B694, 35 (2004).

[3] M. Pepe, Nucl. Phys. Proc. Suppl. 141, 238 (2005).

[4] M. Pepe, PoS (LAT2005), 017 (2005).

[5] M. Engelhardt and H. Reinhardt, Nucl. Phys. B585, 591 (2000).

[6] M. Engelhardt, Nucl. Phys. B585, 614 (2000).

[7] M. Engelhardt, Nucl. Phys. B638, 81 (2002).

[8] G. 't Hooft, Nucl. Phys. B138, 1 (1978).

[9] Y. Aharonov, A. Casher and S. Yankielowicz, Nucl. Phys. B146, 256 (1978).

[10] J. M. Cornwall, Nucl. Phys. B157, 392 (1979).

[11] G. Mack, Phys. Rev. Lett. 45, 1378 (1980).

[12] H. B. Nielsen and P. Olesen, Nucl. Phys. B160, 380 (1979).

[13] L. Del Debbio, M. Faber, J. Greensite and Š. Olejník, Phys. Rev. D 55, 2298 (1997).

[14] L. Del Debbio, M. Faber, J. Giedt, J. Greensite and Š. Olejník, Phys. Rev. D 58, 094501 (1998).

[15] T. G. Kovács and E. T. Tomboulis, Phys. Rev. D 57, 4054 (1998).

[16] P. de Forcrand and M. D’Elia, Phys. Rev. Lett. 82, 4582 (1999).

[17] M. Engelhardt, K. Langfeld, H. Reinhardt and O. Tennert, Phys. Rev. D 61, 054504 (2000).

[18] R. Bertle, M. Engelhardt and M. Faber, Phys. Rev. D 64, 074504 (2001).

[19] J. Greensite, Prog. Part. Nucl. Phys. 51, 1 (2003).

[20] M. Engelhardt, M. Quandt and H. Reinhardt, Nucl. Phys. B685, 227 (2004).

[21] M. Engelhardt, Phys. Rev. D 70, 074004 (2004).

[22] M. Quandt, H. Reinhardt and M. Engelhardt, Phys. Rev. D 71, 054026 (2005).

[23] J. M. Cornwall, Phys. Rev. D 61, 085012 (2000).

[24] M. Engelhardt and H. Reinhardt, Nucl. Phys. B567, 249 (2000).

[25] F. Bruckmann and M. Engelhardt, Phys. Rev. D 68, 105011 (2003).

[26] M. Engelhardt, Phys. Rev. D 73, 034015 (2006).

[27] J. Greensite and Š. Olejník, JHEP 0209, 039 (2002).

[28] M. Engelhardt and B. Sperisen, hep-lat/0610074. 